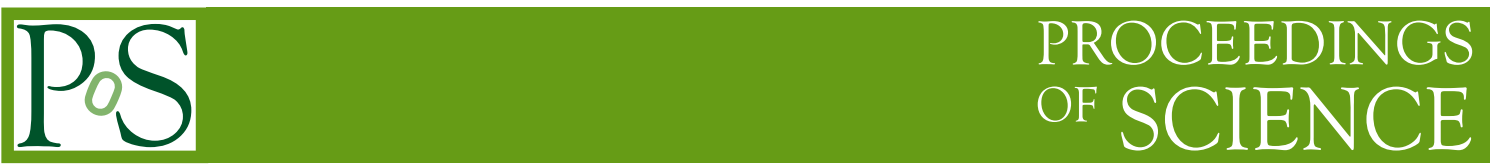

\title{
The Scattering and Neutrino Detector at the LHC
}

\author{
Ettore Zaffaroni ${ }^{a, *}$, on behalf of the SND@LHC collaboration \\ ${ }^{a}$ Ecole Polytechnique Fédérale de Lausanne (EPFL), \\ Lausanne, Switzerland \\ E-mail: ettore.zaffaroni@cern.ch
}

SND@LHC is a compact and stand-alone experiment to perform measurements with neutrinos produced at the LHC in the pseudo-rapidity region of $7.2<\eta<8.6$, complementary to all the other experiments at the LHC.

The experiment will be located $480 \mathrm{~m}$ downstream of the ATLAS interaction point, in the unused TI18 tunnel. The detector is composed of a hybrid system based on an $800 \mathrm{~kg}$ target mass of tungsten plates, interleaved with emulsion and electronic trackers, followed downstream by a calorimeter and a muon system.

The configuration allows to efficiently distinguish between all three neutrino flavours, opening a unique opportunity to probe physics of heavy flavour production at the LHC in the region that is not accessible to ATLAS, CMS and LHCb.

The detector concept is also well suited to searching for Feebly Interacting Particles via signatures of scattering in the detector target.

The data acquisition system is based on a custom board and front-end electronics which operates in a trigger-less fashion, by sending all recorded hits to a central server, where online event building and noise suppression is performed.

The detector is currently being commissioned in the SPS testbeam area and will be installed in the TI18 tunnel by the end of 2021. The first phase aims at operating the detector throughout LHC Run 3 to collect a total of $150 \mathrm{fb}^{-1}$.

\footnotetext{
*** Particles and Nuclei International Conference - PANIC2021 ***

*** 5 - 10 September, $2021 * * *$

$* * *$ Online $* * *$
} 


\section{Introduction}

SND@LHC is a compact experiment, developed to measure neutrinos of all three flavours, produced in the LHC in the pseudo-rapidity range of $7.2<\eta<8.6$. This will allow, together with the FASER $v$ [1] experiment, the first observation of neutrinos produced in a particle collider, in an energy range otherwise inaccessible. The two experiments will measure neutrinos in different angular ranges, where the relative compositions of the various sources are different.

Moreover, SND@LHC can contribute to the search of new physics beyond the Standard Model (BSM) because it is sensitive to Feebly Interacting Particles (FIP) through scattering off atoms in the detector target.

The SND@LHC experiment will be installed in the unused TI18 tunnel, $480 \mathrm{~m}$ downstream of the ATLAS interaction point and shielded by $\sim 100 \mathrm{~m}$ of rock. Preparation works in the site are currently ongoing and the detector will be installed in November 2021.

\section{Physics goals}

Neutrinos allow precise tests of the Standard Model (SM) [2-5] and are a probe for new physics $[6,7]$. The existing measurements of the neutrino cross-section in Figure 1 show that the region between $350 \mathrm{GeV}$ and $10 \mathrm{TeV}$ is currently unexplored [8], as measurements of neutrino interactions in the last decades were mainly performed at low energies. SND@LHC will study neutrinos at higher energies, up to a few $\mathrm{TeV}$.

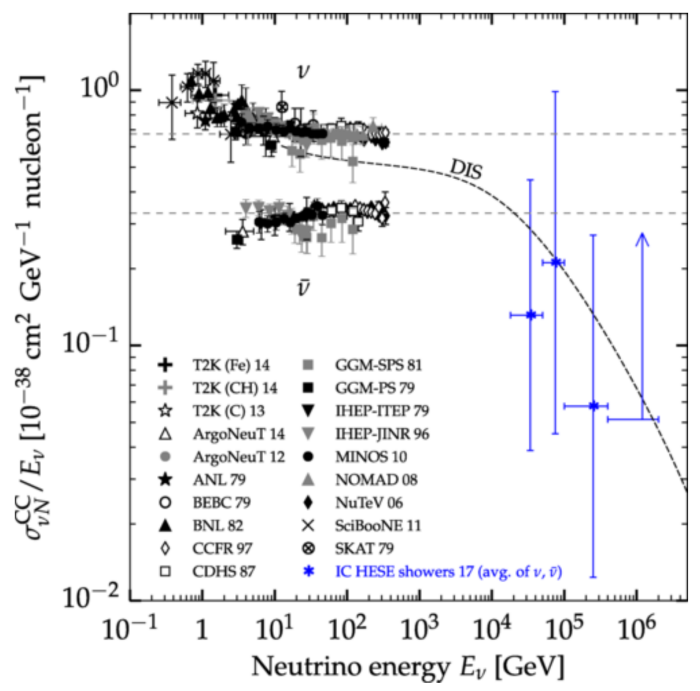

Figure 1: Available measurements of the $v$ and $\bar{v}$ cross-section at different energies. [8]

The SND@LHC experiment has several goals, such as the study of charmed hadrons production in $p p$ collisions at high pseudorapidity, the test of the lepton flavour universality in neutrino interactions and the measurement of the neutral current to charged current cross section (NC/CC) ratio, from which the Weinberg angle can be calculated. The latter is used as an internal consistency check. 
In addition, SND@LHC is capable of performing model-independent direct searches for FIPs by combining the search for a recoil signature with a time-of-flight (TOF) measurement to reject neutrino interactions. A time resolution of the order of $\sim 200 \mathrm{ps}$ allows to disentangle the scattering of massive FIPs and neutrinos, with a significance that depends on the particle mass. [9]

\section{The detector}

The detector, shown in Figure 2, is composed of a target region, followed by a muon identification system. The target region is composed of five Emulsion Cloud Chamber (ECC) walls interleaved with Scintillating Fibres ( $\mathrm{SciFi}$ ) tracker planes. The muon identification system consists of eight iron slabs, each followed by a plane of scintillating bars. In addition, two planes of scintillating bars are placed in front of the target region to act as a veto for charged particles.

All the active detectors are based on scintillators and are read out using Silicon Photomultipliers (SiPMs), with different characteristics based on their application: the SciFi tracker uses finely segmented SiPM arrays, to achieve a space resolution better than $100 \mu \mathrm{m}$, while the other subsystems use larger SiPMs, to achieve a higher efficiency and dynamic range.

The combination of SciFi and the muon detector also acts as a non-homogeneous hadronic calorimeter, with an average length of $9.5 \lambda_{\text {int }}$ ( 8 to $11 \lambda_{\text {int }}$, depending on the position of the neutrino interaction within the target), for the measurement of the energy of the hadronic showers produced in neutrino interactions.

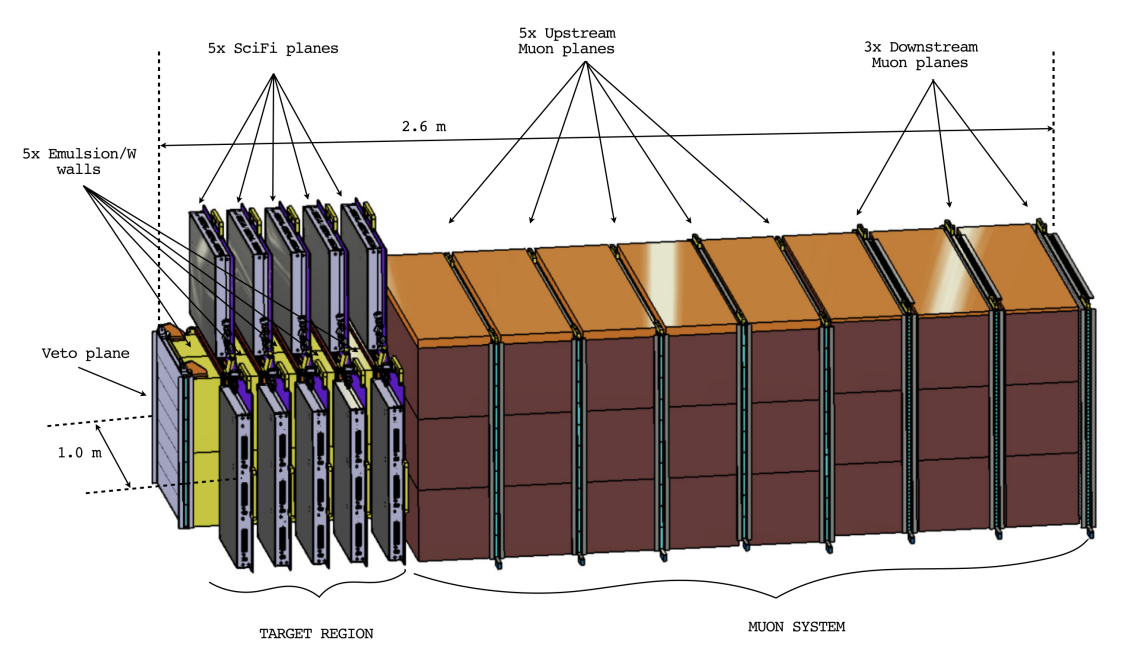

Figure 2: Simplified layout of the SND@LHC. The support mechanics are not shown. [9]

\section{The data acquisition system}

All the active subsystems are read out with the same Data Acquisition (DAQ) electronics, based on a main DAQ board, featuring a Cyclone V FPGA, and up to four front-end (FE) boards, based on the TOFPET2 ASIC, by PETsys. 
The detector utilises a total of 36 DAQ boards, which run synchronously with the LHC bunch crossing clock, received from the Beam Synchronous Timing (BST) system, and delivered to the boards using the Timing, Trigger and Control (TTC) system optical fibres.

The detector is operated in a trigger-less fashion, i.e. all hits recorded by each board are transmitted to the DAQ server. Noise reduction is performed at the front-end level, by setting an appropriate threshold for each channel, and in software, during event building. [9]

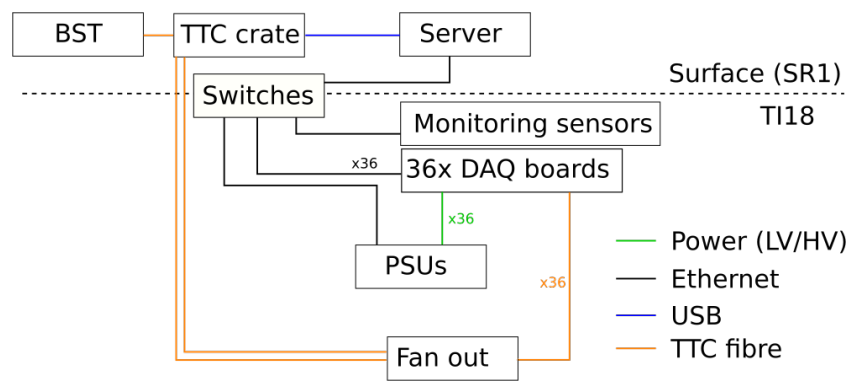

(a)

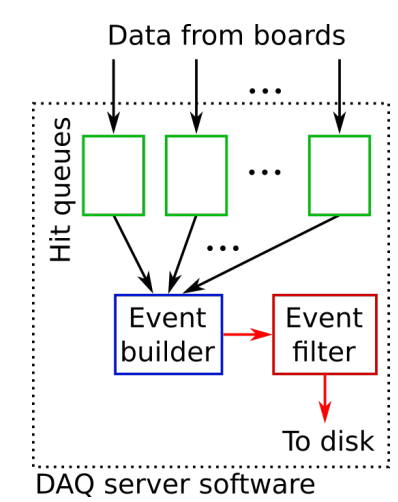

(b)

Figure 3: Scheme of the DAQ system (a) and of the event building (b).

\section{Detector commissioning}

The test and commissioning of the SND@LHC detector is ongoing. Tests of the different sub-systems have been performed separately before moving the detectors to CERN.

As an example, the SciFi tracker has been commissioned at EPFL using cosmic rays. This phase has allowed to have some initial performance determination and to find the best configurations to operate the front-ends. In particular the maximum hit rate that can be sustained by the event builder has been determined to be $\sim 200 \mathrm{kHz}$. An example of two recorded cosmic events is shown in Figure 4.

The subdetectors have been subsequently assembled at CERN, in the SPS testbeam hall, in order to proceed with the commissioning of the full detector. The muon system has been tested with pions of different energies, to calibrate its response as a hadronic calorimeter, and then with muons, together with the SciFi and veto systems. The data analysis is currently ongoing and aims at determining parameters as efficiency, time and spatial resolution, energy response and linearity.

\section{Conclusions}

The SND@LHC detector is a compact experiment optimized to measure the three neutrino flavours and search for physics beyond the Standard Model. It is composed of a veto plane, a target region and a muon system, which also acts as a hadronic calorimeter. It features a trigger-less read-out, with online event building and noise reduction. Its construction and commissioning is underway and it is expected to be installed in the TI18 tunnel at CERN by the end of 2021, in order to start collecting data during the LHC Run 3. 

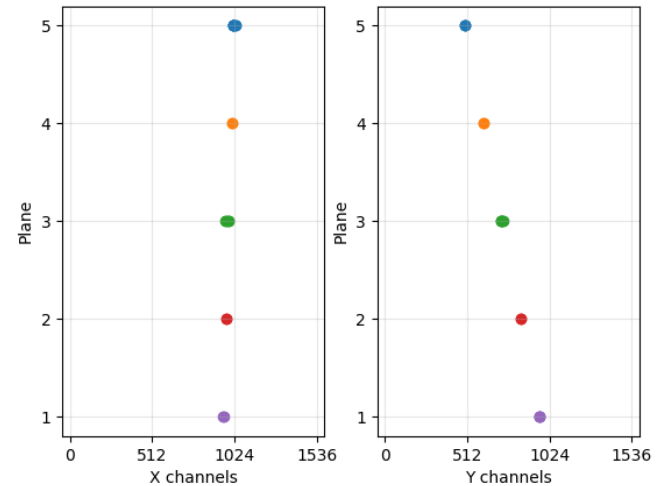

(a)
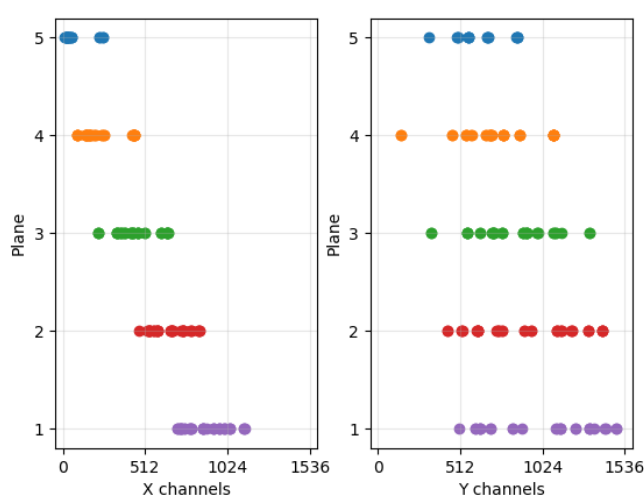

(b)

Figure 4: Two event displays obtained from the commissioning phase using cosmic rays: a muon candidate (a) and a shower candidate (b).

\section{References}

[1] FASER collaboration, Detecting and Studying High-Energy Collider Neutrinos with FASER at the LHC, Eur. Phys. J. C 80 (2020) 61 [1908 . 02310].

[2] CTEQ collaboration, Handbook of perturbative QCD: Version 1.0, Rev. Mod. Phys. 67 (1995) 157.

[3] J.M. Conrad, M.H. Shaevitz and T. Bolton, Precision measurements with high-energy neutrino beams, Rev. Mod. Phys. 70 (1998) 1341 [hep-ex/9707015].

[4] J.A. Formaggio and G.P. Zeller, From eV to EeV: Neutrino Cross Sections Across Energy Scales, Rev. Mod. Phys. 84 (2012) 1307 [1305.7513].

[5] G. De Lellis, P. Migliozzi and P. Santorelli, Charm physics with neutrinos, Phys. Rept. 399 (2004) 227.

[6] D. Marfatia, D.W. McKay and T.J. Weiler, New physics with ultra-high-energy neutrinos, Phys. Lett. B 748 (2015) 113 [1502.06337].

[7] C.A. Argüelles et al., White Paper on New Opportunities at the Next-Generation Neutrino Experiments (Part 1: BSM Neutrino Physics and Dark Matter), 1907.08311.

[8] M. Bustamante and A. Connolly, Extracting the energy-dependent neutrino-nucleon cross section above 10 tev using icecube showers, Phys. Rev. Lett. 122 (2019) 041101.

[9] C. Ahdida et al., SND@LHC - Scattering and Neutrino Detector at the LHC, Tech. Rep. CERN-LHCC-2021-003, LHCC-P-016, CERN, Geneva (Jan, 2021). 